\title{
New "improved" Compton scatter tomography modality for investigative imaging of one-sided large objects
}

\author{
J. Cebeiro ${ }^{a, b}$, M. K. Nguyen ${ }^{b}$, M. A. Morvidone ${ }^{a}$ and A. Noumowéc \\ ${ }^{a}$ Centro de Matemática Aplicada, Universidad Nacional de San Martín, San Martín, Argentina; bequipes de \\ Traitement de I'Information et Systèmes (ETIS), ENSEA/Université de Cergy-Pontoise/CNRS UMR 8051, \\ Cergy-Pontoise, France; ' Laboratoire de Mécanique et Matériaux du Génie Civil (L2MGC), EA 4114/Université \\ de Cergy-Pontoise, Cergy-Pontoise, France
}

\section{ABSTRACT}

In an effort to promote Compton scatter tomography (CST) as an adequate modality for imaging the inner parts of large objects limited in a half-space of $\mathbb{R}^{3}$, we show that the standard CST can be 'improved' in some particular sense by 'doubling' the scanning mechanism. To this end, one needs to record for each position of a detector the scattered flux densities at two energies of scattered radiation, instead of one. Curiously, thanks to geometric inversion, this 'double' scanning may be converted into a transmission scanning on an apparent object, not along a straight line path as usual, but along a folded line in the shape of a $V$-line path. It has been proved that this $V$-line transmission scanning admits a well-defined reconstruction procedure. Therefore, the proposed CST with 'double' scanning, which solves the above imaging inverse problem, shows an 'improved' reconstructed image as compared to the standard one. We present theoretical arguments to support this claim as well as numerical simulations to illustrate its working and its viability.

\section{ARTICLE HISTORY}

Received 22 August 2016

Accepted 4 January 2017

\section{KEYWORDS}

Radon transforms; gamma-ray imaging; Compton scattering; Radon transform on a pair of supplementary circular arcs; $V$-line Radon transform

\section{AMS SUBJECT} CLASSIFICATIONS 44A12; 65J22; 65R10; 68U10

\section{Introduction}

Imaging technology has brought tremendous progress in medical diagnostics as well as in many other fields such as non-destructive surveillance and control, underground exploration, astronomy, homeland security, etc. In most of the cases, objects of interest are of small sizes and imaging equipment blue is designed to fit these object sizes. For very large objects, in particular massive ones but presenting a very large flat surface, there is a need of special imaging devices which can accommodate such structures. Existing imaging systems are based on acoustic waves and electromagnetic waves propagation properties. One may cite e.g. Sonar imaging or Ground Radar Imaging, which deliver undersea images of sunken ships or underground images of buried pipes, etc. Image reconstruction is based mostly on information given by backscattered waves and this procedure requires ad-hoc algorithms.

Moreover, sound and electromagnetic waves have limited penetration in dense matter. To reveal finer structure there is a need to penetrate further in dense matter with shorter 
wavelengths. This is why ionizing radiation is appropriate for probing the inner structure of dense matter, which is proper to industrial objects as well as rocky medium or concrete. The interest in using ionizing radiation to explore the inner structure of objects has been proposed a long time ago and has known in the recent years a renewed interest. Ionizing radiation is in fact the ideal tool to prospect the inner structure of matter because of its strong penetrating property. However, information on the probed object matter can only be retrieved if one manages to record the outgoing radiation, which has undergone an interaction inside matter. Of course one may register backscattered for acoustic as well as for electromagnetic waves. Several concepts for gathering backscattered radiation have been proposed in the past, see e.g. [1-3]. Here there is a possibility of recover scattered waves which are far more versatile. In particular in the energy range around $150 \mathrm{keV}$, the only relevant radiation interaction is Compton scattering. This is why the concept of Compton imaging was initiated and developed in last few decades. We shall not recall the early steps of Compton imaging and refer the reader to [4], but give some references on recent interests in Compton imaging for historical object research and conservation [5], land mine detection [6], agricultural measurements [7], battery functioning [8], nondestructive testing [9] and soil studies [10].

One of the main advantages of Compton scattering imaging over X-ray transmission imaging is its high sensitivity to the local electron density, even for materials with high 20 X-ray absorption coefficients. This is because Compton scattering imaging measures the $\mathrm{X}$-ray intensity from a local probing volume, while X-ray transmission imaging records the total absorption along the X-ray path. This is very beneficial for large objects.

To this end, Compton scatter tomography (CST) has been advocated for investigating the hidden structure of one-sided large objects $[11,12]$, when this investigation is done slice by slice on this object. However, it has not yet emerged as a widely used modality in this field mainly due to poor sensitivity of image reconstruction. In this work, we reanalyse the properties of this classical CST modality to explain its functioning principle and then propose a new modality which, in principle exhibits an enhanced image reconstruction, potentially more efficiency in the study of 'beyond the wall' structure of large one-sided objects or half-space extended medium. In CST, data acquisition can be done by a detector located on the same side of the object under investigation: this is not the case in conventional transmission tomography. CST is expected to give high contrast, high spatial resolution and stoichiometric independent images of two-dimensional slices of objects. CST is appropriate for imaging continuing flow of liquids in pipes, solid propellant rocket engines, ships hulls, aircraft parts, large castings, etc. in particular when the need of obtaining access to both sides of the structure is denied. This is really advantageous at a time when new generation of position- and energy-sensitive radiation detectors have appeared. Throughout the years, there has been substantial progress in development of CST modalities [4]. However in this work, we shall concentrate on a new modality, which was proposed recently [13] but not assessed numerically as far as feasibility and realizability are concerned. The purpose of this paper is to close this gap by giving detailed computation on image formation as well as reconstruction and image quality evaluation.

As always, an imaging modality relies on a mathematical tool which supports its image formation and provides a way to reconstruct an image. In conventional computed 45 tomography, this role was played by the well-known classical Radon transform. For CST, it has been established that a variety of Radon transforms on circular arcs forms the support 
for image formation when higher order radiation scattering is not taken into account. Moreover, these generalized Radon transforms are shown to be invertible paving the way to image reconstruction in CST. Here we shall investigate the imaging capabilities of a newly proposed Radon transform, which is defined on a pair of circular arcs (instead of a single circular arc), introduced in [13]. Its invertibility is shown to be transferred to the invertibility of the so-called Radon transforms on $V$-lines by geometric inversion of the coordinate variables. This type of transfer has been known for a long time for the Radon transform on circles intersecting a fixed point in a plane, which become simply the classical Radon transform on straight lines of the same plane [14]. The $V$-line is the geometric object which consists of two half-lines ending at a vertex-point on an horizontal axis and disposed symmetrically around the vertical axis at the vertex-point and making a variable angle with it. We adopt this terminology in order to distinguish it from the so-called Radon transform on broken lines introduced by [15], which is defined on the set of geometric figures made of two half-lines making a fixed angle between them and of arbitrary location and orientation in the plane.

The paper is structured as follows. The fundamentals of Compton scattering tomography, in particular the classical CST modality proposed by S. J. Norton in 1994 [12] are reviewed in some details, in which we recall the Compton scattering phenomena and its properties. We also recast the conventional CST modality of S. J. Norton in the framework of the Radon transform on half-lines and describe its solution, which solves the inverse problem of CST imaging. The next Section 3 concerns the new CST modality. We show how the scanning on a pair of supplementary circular arcs is beneficial for imaging an object via conversion into a transmission scanning problem on a $V$-line. We also demonstrate theoretically why the reconstruction scheme yields an improved' result as compared to the standard one. A major point is the introduction of the inversion procedure as a filtered back projection (FBP), which is widely used in conventional tomography and provides an elegant way to deal with divergencies in coordinate space. A working algorithm is given to show the working of the numerical simulations in Section 5. The numerical simulations results, with a careful analysis of image quality under different working conditions, illustrate the feasibility and viability of this new CST imaging modality. The paper ends with a conclusion and some future directions of investigation.

\section{Compton scattering tomography: a review}

Compton scattering tomography (CST) was introduced in the early 80s, see e.g. [16], but it was S. . Norton who gave a full theoretical treatment in 1994 [12]. A threedimensional object is illuminated by a penetrating radiation external point source $S$ of calibrated intensity. Since tomography considers only one planar slice of this object, this point source will be placed between two collimator plates, which are parallel to the slice and separated by a small distance to restrict the emitted radiation to a plane in fact a thin slice, before it irradiates the object situated beyond these collimator plates. First-order scattered radiation of specific energy is registered by a point detector $D$ placed at a distance $\xi$ from the source, also located in between the collimator plates. The detector is assumed to be highly energy sensitive and of sufficiently small size (because the intensity of scattered radiation is low), in order to be able to generate a very large amount of data. In the present work, radiation attenuation is not taken into account as we concentrate on the working 


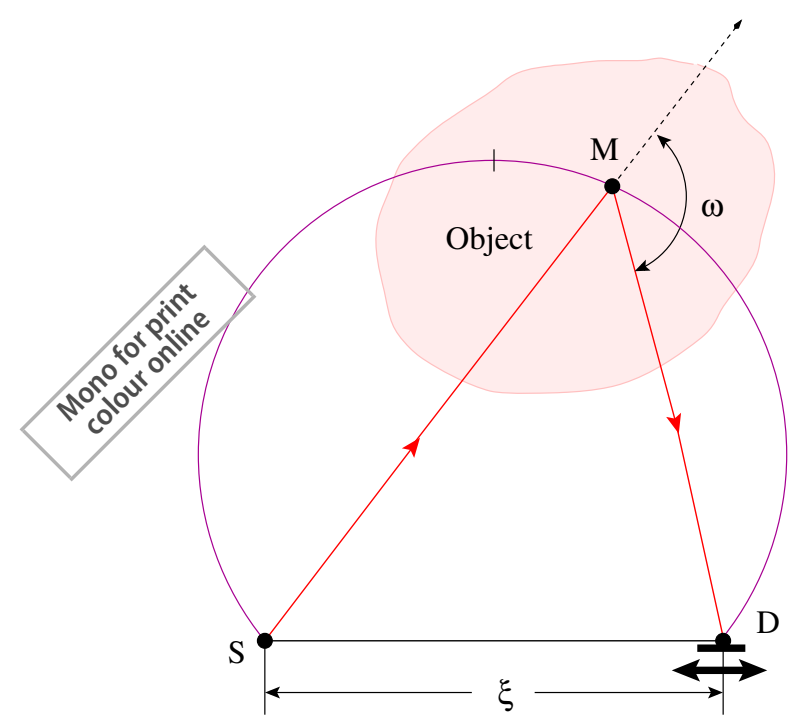

Figure 1. Classical CST.

principle. It is expected that radiation attenuation is important before and after scattering inside matter and should be corrected. However, it is known that including attenuation in such processes usually does not allow an analytic solution. Even in the case of simple emission imaging the inclusion of arbitrary attenuation was only exactly solved in 2002 [17]. However, a constant attenuation may be manageable, a kind of pre-correction may be performed such as in [18] and shall be deferred to a future investigation. The claim of CST is that the totality of such measurement is sufficient to reconstruct an image of the inner part of the object, which consists of the electron density of its interior.

Figure 1 summarizes the working of this modality. As penetrating radiation consists of X-(or -gamma) rays, an object, represented by the shaded area is subjected to the illuminating radiation carrying an original energy $E_{0}$ of an isotropic source $S$ placed at the origin of a Cartesian coordinate system. A point-like detector at site $D$, at a distance $\xi$ from $S$, with a flat collimator under it to allow only the recording of scattered radiation of scattered energy $E(\omega)$ (and not of energy $E(\pi-\omega)$ ), moves on the axis $S D$ and registers a radiation flux density at a single Compton scattered energy $E(\omega)$, which is related to the scattering angle $\omega$ by the so-called Compton relation

$$
E(\omega)=\frac{E_{0}}{1+\frac{E_{0}}{m c^{2}}(1-\cos \omega)},
$$

where $m c^{2}$ represents the energy of the electron at rest $(511 \mathrm{keV})$. Inspection shows that the totality of radiation flux density at a detecting site $D$ situated at a distance $\xi$ from $S$ is due to all scattering sites $M$ located on a circular arc starting from $S$ and ending at $D$, subtending an inscribed angle $(\pi-\omega)$, which is constant for each measurement. The kinematics of Compton scattering (see Figure 2) is encoded in the Klein-Nishina differential cross section

$$
\frac{\mathrm{d} \sigma}{\mathrm{d} \Omega}=\pi r_{e}^{2} P(\omega)
$$




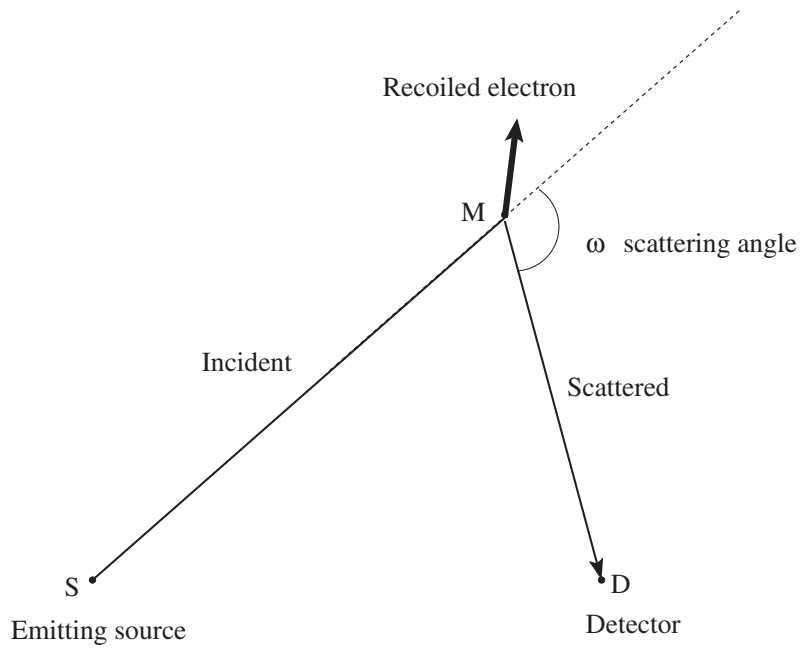

Figure 2. Compton scattering.

where $\sigma$ is the Compton scattering cross-section, $r_{e}$ is the classical electron radius and $P(\omega)$ is the Klein-Nishina probability to be scattered in the solid angle $\Omega$. The expression of $P(\omega)$ is given in [19].

Let us introduce a Cartesian coordinate system $x S y$ centred at $S$ with $D$ on the $x$-axis. The registered scattered radiation flux density at $D$ is essentially the integral of the electric charge density of the object on a circular arc, which has a radius $\rho=\frac{\xi}{2 \sin \omega}$ and a centre $\Omega$ at $\left(\frac{\xi}{2}, \frac{\xi}{2 \tau}\right)$, where $\tau=\tan \omega$. Let $f(x, y)$ be this electric charge density. To preserve the clarity of the exposition, we shall neglect propagation photometric effects and radiation attenuation in matter, as stated before. Let us assume that the source $S$ emits isotropically 10 radiation of energy $E_{0}$ and that the point detector $D$ has also spherical symmetry. The amount of radiation registered at $D$ is then of the form

$$
\mathcal{R}_{C} f(\xi, \omega)=K(\omega) \int_{C} f(x, y) \mathrm{d} s_{C},
$$

where the $\omega$-dependent factor $K(\omega)$ contains besides the Klein-Nishina probability $P(\omega)$ all other kinematic factors of the scattering at site $M=(x, y), C$ is the circular arc SMD and its arc element $\mathrm{d} s_{C}$. So the quantity $\mathcal{R}_{C} f(\xi, \omega) / K(\omega)$, from now on called the renormalized data, is the integral of an unknown function $f(x, y)$ on a set of circular arcs subtending an inscribed angle $(\pi-\omega)$ starting at the source point $S$ and ending at the detection point $D$. It is a function of $(\xi, \omega) \in \mathbb{R} \times[0, \pi]$. The problem is now how to reconstruct the electric charge density $f(x, y)$ from the set of measurements $\mathcal{R}_{C} f(\xi, \omega)$. There are several possible 20 approaches.

S. J. Norton has argued that if the object is 'above' the axis $S x$, there is no harm to carry out the integration on the full circle, which is of variable centre in the plane but always passing through the fixed point $S$. $\mathcal{R}_{C} f(\xi, \omega) / K(\omega)$ is thus the Radon transform of $f(x, y)$ on the set of circles of the plane intersecting a fixed point $S$. But already in 1965, A. M. Cormack has studied such a Radon transform [14] and he has also given the corresponding inverse formula. ${ }^{1}$ This situation is applicable whenever the object has a compact support. 


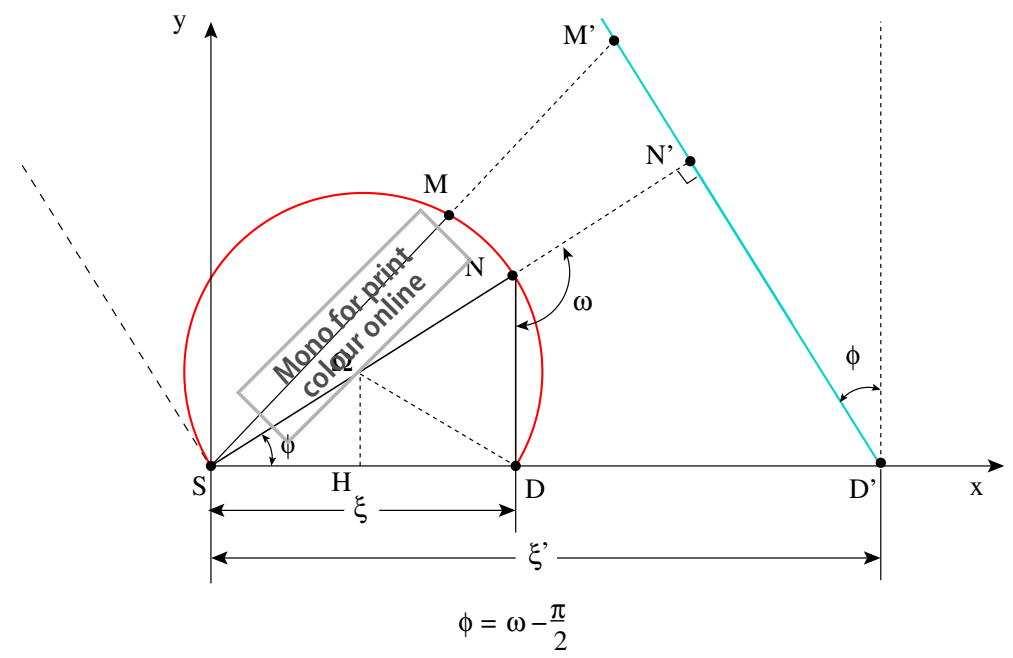

Figure 3. Geometric inversion of a circular arc.

Numerical implementation of this procedure has been given recently in [18] using the so-called circular component expansion.

Recently, it has been shown in [13] that the Radon transform of a function $f(x, y)$ on the above circular arcs can be converted, by geometric inversion of centre $S$ and inversion circle of radius $R$ into a Radon transform on half-lines contained in upper half plane of the line $S D$, not for $f(x, y)$ but for a modified function $g_{R}\left(x^{\prime}, y^{\prime}\right)$, given by

$$
g_{R}\left(x^{\prime}, y^{\prime}\right)=\frac{R^{2}}{x^{\prime 2}+y^{\prime 2}} f\left(\frac{R^{2} x^{\prime}}{x^{\prime 2}+y^{\prime 2}}, \frac{R^{2} y^{\prime}}{x^{\prime 2}+y^{\prime 2}}\right),
$$

where the transform by geometric inversion of a point $M=(x, y)$ in the $x S y$ plane is $M^{\prime}=\left(x^{\prime}, y^{\prime}\right)$ given by

$$
\left(x^{\prime}, y^{\prime}\right)=\frac{R^{2}}{x^{2}+y^{2}}(x, y) \text {. }
$$

Figure 3 shows how the circular arc SMD is converted by this geometric inversion into the half-line $D^{\prime} M^{\prime}$, such that

$$
\xi^{\prime}=\frac{R^{2}}{\xi}, \quad \text { and } \quad S M^{\prime}=\frac{R^{2}}{S M} .
$$

The circular arc SMD subtends an angle $(\pi-\omega)$. This arc is transformed into a half-line starting at site $D^{\prime}$ at a distance $\xi^{\prime}$ from $S$ on the axis $S x$. This half-line makes an angle $\phi=(\omega-\pi / 2)$ with the vertical direction. This is so because the line $S N N^{\prime}$ going through the centre $\Omega$ of the circle $S M N D$ is perpendicular to the half-line $D^{\prime} N^{\prime} M^{\prime}$.

Note that in Figure 3, $\xi>0$ and the scattering angle $\omega$ is chosen to be larger than $\pi / 2$, hence $\phi$ is positive. The equation of the circular $\operatorname{arc} S M D$, with centre at $\Omega=\left(\frac{\xi}{2}, \frac{\xi}{2 \tau}\right)$ is

$$
\left(x-\frac{\xi}{2}\right)^{2}+\left(y-\frac{\xi}{2 \tau}\right)^{2}=\left(\frac{\xi}{2 \sin \omega}\right)^{2}, \text { and } y>0 \text {. }
$$




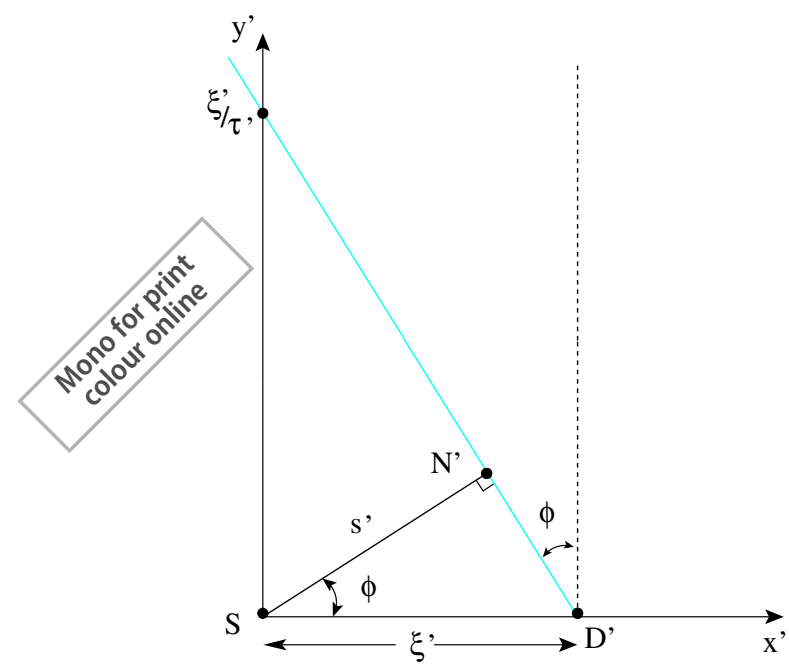

Figure 4. Half-line of equation $\xi^{\prime}-x^{\prime}-y^{\prime} \tau^{\prime}=0$ with $y^{\prime}>0$ used in $\mathcal{R}^{\natural}$ Radon transform.

Now using Equation (5), one gets the equation of a half-line

$$
\xi^{\prime}-x^{\prime}-y^{\prime} \tau^{\prime}=0, \quad \text { and } \quad y^{\prime}>0
$$

where $\tau^{\prime}=\frac{1}{\tau}$.

Let us denote the Radon transform on half-lines by $\mathcal{R}^{\natural}$, see [13]. Then for a function $h\left(x^{\prime}, y^{\prime}\right)$, its integral on the half-line of Equation (8) is (see Figure 4)

$$
\mathcal{R}^{\natural} h\left(\xi^{\prime}, \tau^{\prime}\right)=\int_{0}^{\infty} \mathrm{d} y^{\prime} \sqrt{1+\tau^{\prime 2}} h\left(\xi^{\prime}-y^{\prime} \tau^{\prime}, y^{\prime}\right),
$$

where $s^{\prime}=S N^{\prime}=\xi^{\prime} \cos \phi=\xi^{\prime} \sin \omega$, with $\left(\xi^{\prime}, \tau^{\prime}\right) \in \mathbb{R}^{2}$.

Now by identifying the circular aro Radon data $\mathcal{R}_{C} f(\xi, \omega) / K(\omega)$ to the data of a Radon transform on half-lines $\mathcal{R}^{\natural}$ according to

$$
\frac{\mathcal{R}_{C} f(\xi, \omega)}{K(\omega)}=\mathcal{R}^{\natural} g_{R}\left(\xi^{\prime}, \tau^{\prime}\right),
$$

we can use the inyerse formula for the Radon transform of half-lines of the plane (given in [13]), applied to the data $\mathcal{R}^{\natural} g_{R}\left(\xi^{\prime}, \tau^{\prime}\right)$ to reconstruct $g_{R}\left(x^{\prime}, y^{\prime}\right)$, or

$$
g_{R}\left(x^{\prime}, y^{\prime}\right)=-\frac{1}{2 \pi^{2}} H\left(y^{\prime}\right) \int_{\mathbb{R}^{2}} \frac{\mathrm{d} \xi^{\prime} \mathrm{d} \tau}{\sqrt{1+\tau^{2}}} \frac{1}{\xi^{\prime}-x^{\prime}-y^{\prime} \tau^{\prime}} \frac{\partial}{\partial \xi^{\prime}} \mathcal{R}^{\natural} g_{R}\left(\xi^{\prime}, \tau^{\prime}\right),
$$

where $H\left(y^{\prime}\right)$ is the Heaviside function of $y^{\prime} . f(x, y)$ is recovered through

$$
f(x, y)=\frac{R^{2}}{x^{2}+y^{2}} g_{R}\left(\frac{R^{2} x}{x^{2}+y^{2}}, \frac{R^{2} y}{x^{2}+y^{2}}\right) .
$$

Note that the parameter $R$ drops out at the end of the reconstruction of $f(x, y)$. 


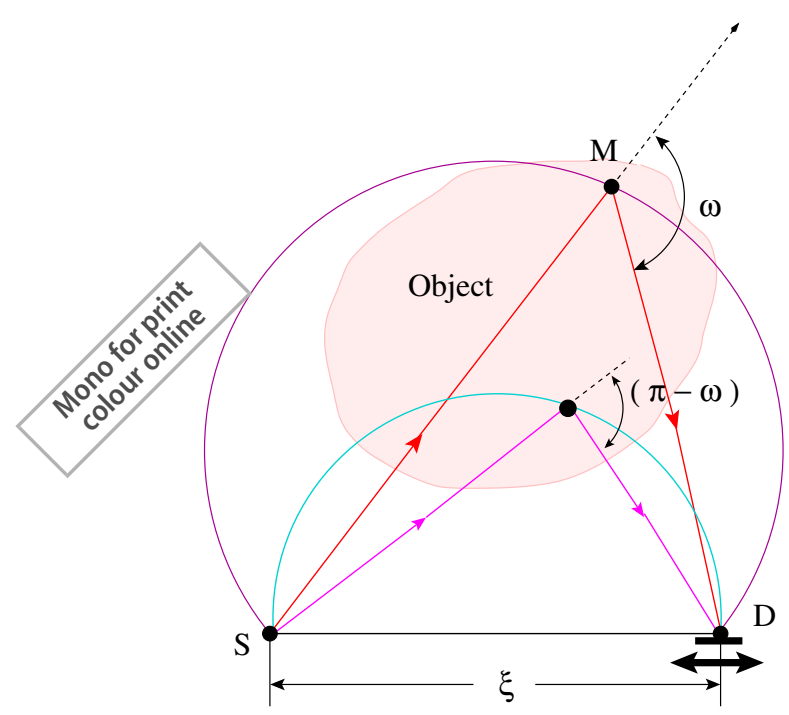

Figure 5. Double supplementary arcs CST.

\section{New 'improved' modality for CST}

In imaging science, the aim is to obtain the most appropriate imaging modality. For CST, following a recent suggestion in [13], which consists in gathering measured data not only on one circular arc but on two circular arcs, see Figure 5, we undertake to explore the performances of this new modality. Previously, on a circular arc SMD subtending an angle $(\pi-\omega)$ and based on a chord $S D=\xi$, the integration path of the electron density has a length which can be at most equal to $\omega \xi / \sin \omega$. By adding a new circular arc $S N D$ to gather data one may expect a longer integration path. In fact for reasons that become clear later, we shall use a second circular arc SND which subtends an angle $\omega$ and based on the same chord $S D=\xi$. Then the total integration path used for data collection will be always the full circumference of length $\pi \xi / \sin \omega$, which is clearly larger than $\omega \xi / \sin \omega$, since $0<\omega<\pi$. Intuitively, one may suspect that this data acquisition would be more beneficial to image reconstruction. But this must be checked.

To simplify notations, we shall denote generically the circular arc SMD (resp. SPD) by $C$ (resp. $C^{\prime}$ ) in Figure 5. So at site $D$, two measurements of scattered radiation at energy $E(\omega)$ and at energy $E(\pi-\omega)$ will be made and recorded according to

$$
\mathcal{R}_{C} f(\xi, \omega)=K(\omega) \int_{C} f(x, y) \mathrm{d} s_{C}, \quad \text { and } \quad \mathcal{R}_{C^{\prime}} f(\xi, \omega)=K(\pi-\omega) \int_{C^{\prime}} f(x, y) \mathrm{d} s_{C^{\prime}}
$$

Note that $\mathrm{d} s_{C}=\mathrm{d} s_{C^{\prime}}$. Now if one sums up the renormalized data after division by the corresponding $K(\omega)$ and $K(\pi-\omega)$ factors

$$
\frac{\mathcal{R}_{C} f(\xi, \omega)}{K(\omega)}+\frac{\mathcal{R}_{C} f(\xi, \pi-\omega)}{K(\pi-\omega)}=\mathcal{R}_{C C^{\prime}} f(\xi, \omega)
$$




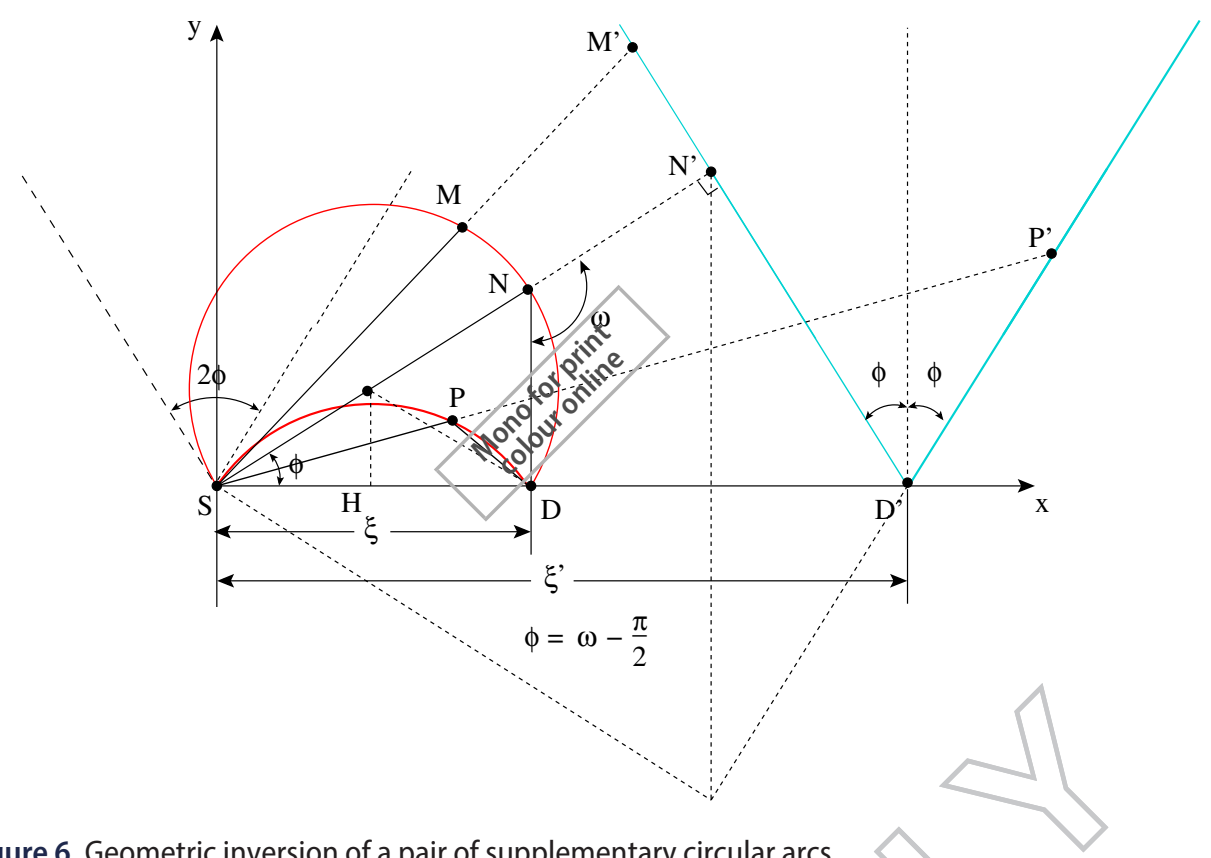

Figure 6. Geometric inversion of a pair of supplementary circular arcs.

as $\mathcal{R}_{C C^{\prime}} f(\xi, \omega)$, which is the data of a Radon transform on a pair of supplementary circular arcs $C C^{\prime}$ of a function $f(x, y)$, the problem is now how to obtain $f(x, y)$ from this data $\mathcal{R}_{C C^{\prime}} f(\xi, \omega)$.

Following the idea of Section 2, we may apply the same geometric inversion to the pair of circular arcs $S M D$ (or $C$ ) and SPD (or $C^{\prime}$ ), each of which subtends, respectively, the angle $(\pi-\omega)$ and $\omega$, see Figure 6 . Each of the circular arcs will be transformed into a half-line starting at site $D^{\prime}$ at a distance $\xi^{\prime}=R^{2} / \xi$ from $S$ on the axis $S x$. Each of these half-lines makes an angle $\phi=(\omega-\pi / 2)$ with the vertical direction. This is so because the line $S N N^{\prime}$ going through the centre of the circle $S M N D$ is perpendicular to the half-line $D^{\prime} N^{\prime} M^{\prime}$. The resulting figure $N^{\prime} D^{\prime} P^{\prime}$ is a so-called $V$-line as stated in [13].

Thus geometric inversion turns this data into the data for a Radon transform on $V$-lines for the function $g_{R}\left(x, y^{\prime}\right)$ encountered before in Equation (11). Let $\mathcal{R}_{V} g_{R}\left(\xi^{\prime}, \tau^{\prime}\right)$ be the Radon transform on $V$-lines of $g_{R}\left(x^{\prime}, y^{\prime}\right)$. Then we may identify

$$
\mathcal{R}_{C C^{\prime}} f(\xi, \omega)=\mathcal{R}_{V} g_{R}\left(\xi^{\prime}, \tau^{\prime}\right)
$$

with $\tau^{\prime}=\cot \omega$. Recall that the $V$-line transform of $g_{R}\left(x^{\prime}, y^{\prime}\right)$ is given by

$$
\mathcal{R}_{V} g_{R}\left(\xi^{\prime}, \tau^{\prime}\right)=\sqrt{1+\tau^{\prime 2}} \int_{0}^{\infty} \mathrm{d} y^{\prime}\left\{g_{R}\left(\xi^{\prime}-y^{\prime} \tau^{\prime}, y^{\prime}\right)+g_{R}\left(\xi^{\prime}+y^{\prime} \tau^{\prime}, y^{\prime}\right)\right\} .
$$

In Figure 7, is represented the $V$-line on which a function is integrated and $s^{\prime}=\xi^{\prime} \cos \phi=$ $\xi^{\prime} \sin \omega$ as before.

Knowing that the Radon transform on $V$-lines is invertible (see [13]), we can write down its inverse formula, in which we put the data collected from $\mathcal{R}_{C C^{\prime}}$, the Radon transform on double circular arcs as transformed by Equation (14) 


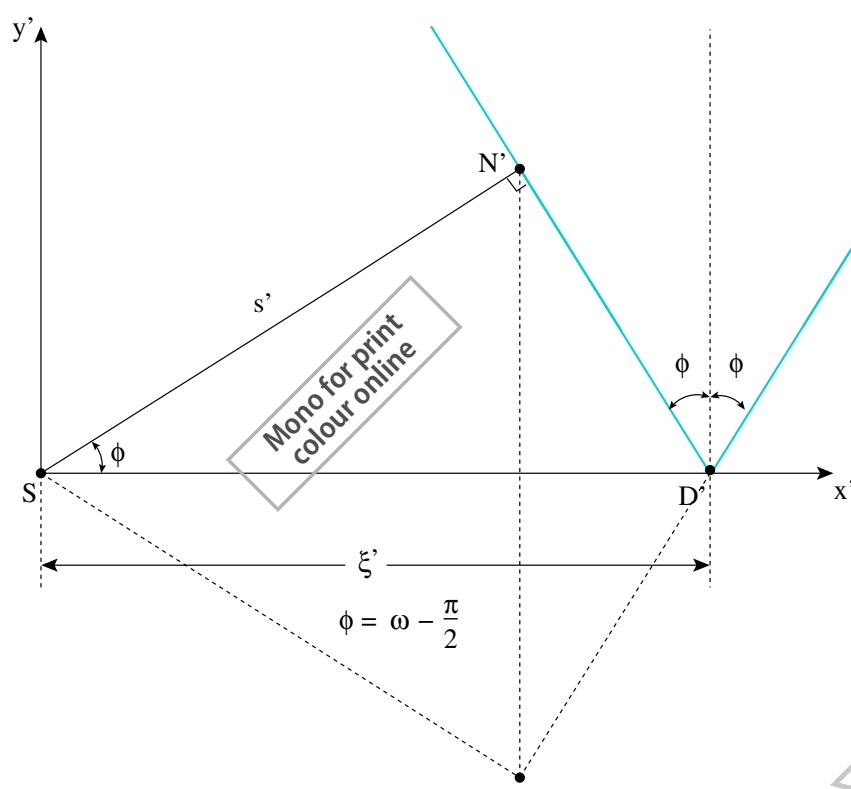

Figure 7. $V$-line of equation $\left|x^{\prime}-\xi^{\prime}\right|=y^{\prime} \tau^{\prime}$ used in $\mathcal{R}_{V}$ Radon transform.

$$
\begin{gathered}
g_{R}\left(x^{\prime}, y^{\prime}\right)=-\frac{1}{2 \pi^{2}} \int_{0}^{\infty} \frac{\mathrm{d} \tau^{\prime}}{\sqrt{1+\tau^{\prime 2}}} \\
\int_{\mathbb{R}} \mathrm{d} \xi^{\prime}\left(\frac{1}{\xi^{\prime}-x^{\prime}-y^{\prime} \tau^{\prime}}+\frac{1}{\xi^{\prime}-x^{\prime}+y^{\prime} \tau^{\prime}}\right) \frac{\partial}{\partial \xi^{\prime}} \mathcal{R}_{C C^{\prime}} f\left(\frac{R^{2}}{\xi^{\prime}}, \omega\right) .
\end{gathered}
$$

The electron density $f(x, y)$ is finally recovered as before by Equation (12).

At this point it is natural to ask what differentiates the two CST modalities? (i.e. the one using a single scanning circular arc and the other using a pair of supplementary scanning circular arcs). Or more precisely, what differentiates their imaging integral transforms which are the Radon transforms, respectively, on one scanning circular arc and on two scanning supplementary circular arcs? To answer this question, it may be simpler to look at their geometric inverse partners, which are, respectively, the half-line Radon transform and the $V$-line Radon transform. These Radon transforms may be viewed as supporting apparent emission imaging problems in the upper half part of a plane (see Figure 3 in [13] and Figure 3 in [20]). Therefore we shall discuss the relation connecting the half-line Radon transform to the $V$-line Radon transform in the upper part of a plane and then extrapolate the conclusion to the two CST modalities.

\section{A relation between the half-line Radon transform and the $\boldsymbol{V}$-line Radon transform}

We recall the essential properties of $\mathcal{R}^{\natural}$ (resp. $\mathcal{R}_{V}$ ) the half-line (resp. $V$-line) Radon transform and refer the reader to [13]. In this section, we use the standard Cartesian coordinate system $(x O y)$, since the discussion is intrinsically independent of the previous sections. 


\subsection{Half-line Radon transform}

The $\mathcal{R}^{\natural}$ transform of an integrable function $f(x, y)$ in $\mathbb{R}^{2}$ is defined as

$$
\mathcal{R}^{\natural} f(\xi, \tau)=\sqrt{1+\tau^{2}} \int_{0}^{\infty} \mathrm{d} y f(\xi-y \tau, y),
$$

where $(\xi, \tau) \in \mathbb{R}^{2}$. Thus $\mathcal{R}^{\natural} f(\xi, \tau)$ is a function defined in $\mathbb{R}^{2}$, obtained by integrating $f(x, y)$ on the half-line of equations $x=\xi-y \tau$ with $y>0$. Its inversion is given by the reconstruction formula (see [13])

$$
f(x, y)=-\frac{1}{2 \pi^{2}} H(y) \int_{\mathbb{R}^{2}} \frac{\mathrm{d} \tau \mathrm{d} \xi}{\sqrt{1+\tau^{2}}} \frac{1}{\xi-x-y \tau} \frac{\partial}{\partial \xi} \mathcal{R}^{\natural} f(\xi, \tau) .
$$

This formula resembles the reconstruction formula for the standard Radon transform except for the Heaviside function $H(y)$, which means that the only data gathered is in the upper plane. Thus, one can only reconstruct the function in the upper plane. A priori, no evident symmetry exists and no extension to the lower part of the plane is available. Only the part of $f(x, y)$ for $y>0$ is reconstructed and nothing can be said on $f(x, y)$ for $y<0$.

\subsection{V-line Radon transform}

The $V$-line Radon transform was defined originally as the integral transform which assigns to a function $f(x, y)$ its integral on a standing $V$-line with vertex on the $O x$-axis and opening angle $\omega$. This $V$-line figure is in fact a two-dimensional version of a circular cone with vertical axis in $\mathbb{R}^{3}$. Recently many variants of this so-called $V$-line Radon transform have appeared in the literature, see [15,21-24]. In a Cartesian coordinate system with origin $O$, the equation of the $V$-line is $|x-\xi|=y \tau$. The vertex is on the $O x$ axis at abscissa $\xi$. The

20 slope of the two branches with respect to the vertical direction is given by $\pm \tau$. Thus the transform $\mathcal{R}_{V} f$ of an function $f$ is

$$
\mathcal{R}_{V} f(\xi, \tau)=\sqrt{1+\tau^{2}} \int_{0}^{\infty} \mathrm{d} y\{f(\xi-y \tau, y)+f(\xi+y \tau, y)\}
$$

with $0<\tau<\infty$. Again we see that $\mathcal{R}_{V} f$ 'sweeps' the upper half-plane $y>0$ as $\mathcal{R}^{\natural}$.

Lemma 4.1: $\mathcal{R}_{V} f(\xi, \tau)$, although defined for $0<\tau<\infty$, is invariant under $\tau \rightarrow-\tau$. Hence $\mathcal{R}_{V} f(\xi, \tau)$ is extendable into $\tau<0$ by

$$
\mathcal{R}_{V} f(\xi, \tau)=\mathcal{R}_{V} f(\xi,-\tau)
$$

It is a symmetric function of $\tau$.

Moreover, in view of the definition of $\mathcal{R}^{\natural}$, we have also the lemma

Lemma 4.2: The V-line Radon transform data is related to the half-line Radon data by

$$
\mathcal{R}_{V} f(\xi, \tau)=\mathcal{R}^{\natural} f(\xi, \tau)+\mathcal{R}^{\natural} f(\xi,-\tau) .
$$

The $\tau$-symmetry of $\mathcal{R}_{V} f(\xi, \tau)$ is now explicit. 
For the $V$-line Radon transform, a function $f(x, y)$ is reconstructed from its data $\mathcal{R}_{V} f(\xi, \tau)$ via the formula

$$
f(x, y)=-\frac{1}{2 \pi^{2}} \int_{0}^{\infty} \frac{\mathrm{d} \tau}{\sqrt{1+\tau^{2}}} \int_{-\infty}^{\infty} \mathrm{d} \xi\left(\frac{1}{\xi-x-y \tau}+\frac{1}{\xi-x+y \tau}\right) \frac{\partial}{\partial \xi} \mathcal{R}_{V} f(\xi, \tau) .
$$

Note the $\tau$-symmetry of the integrand under the integral sign.

Then it is legitimate to ask whether it is possible to express the reconstruction formula of the $V$-line Radon transform in terms of the reconstruction formula of the half-line Radon transform.

This is the content of the next theorem

Theorem 4.3: When applied to the data $\mathcal{R}_{V} f(\xi, \tau)$, the following two inverse integral operators

$$
\left(\mathcal{R}_{V}(x, y)\right)^{-1}=-\frac{1}{2 \pi^{2}} \int_{0}^{\infty} \frac{\mathrm{d} \tau}{\sqrt{1+\tau^{2}}} \int_{-\infty}^{\infty} \mathrm{d} \xi\left(\frac{1}{\xi-x-y \tau}+\frac{1}{\xi-x+y \tau}\right) \frac{\partial}{\partial \xi},
$$

and

$$
\left(\mathcal{R}^{\natural}(x, y)\right)^{-1}=-\frac{H(y)}{2 \pi^{2}} \int_{\mathbb{R}^{2}} \frac{\mathrm{d} \tau \mathrm{d} \xi}{\sqrt{1+\tau^{2}}} \frac{1}{\xi-x-y \tau} \frac{\partial}{\partial \xi},
$$

give the same result.

15 Proof: Start with Equation (23), and use the $\tau$-symmetry to extend the $\tau$-integration to $\mathbb{R}$,

$$
f(x, y)=-\frac{1}{4 \pi^{2}} \int_{-\infty}^{\infty} \frac{\mathrm{d} \tau}{\sqrt{1+\tau^{2}}} \int_{-\infty}^{\infty} \mathrm{d} \xi\left(\frac{1}{\xi-x-y \tau}+\frac{1}{\xi-x+y \tau}\right) \frac{\partial}{\partial \xi} \mathcal{R}_{V} f(\xi, \tau)
$$

It is the sum of two integrals, the first one with a denominator $(\xi-x-y \tau)$. Rewrite the second one with a denominator $(\xi-x+y \tau)$ in terms of $\tau^{\prime}=-\tau$, then using Equation (21) it becomes

$$
-\frac{1}{4 \pi^{2}} \int_{-\infty}^{\infty} \frac{\mathrm{d} \tau^{\prime}}{\sqrt{1+\tau^{\prime 2}}} \int_{-\infty}^{\infty} \mathrm{d} \xi \frac{1}{\xi-x-y \tau^{\prime}} \frac{\partial}{\partial \xi} \mathcal{R}_{V} f\left(\xi, \tau^{\prime}\right)
$$

Putting together and relabel $\tau$ by $\tau$, we get

$$
f(x, y)=-\frac{1}{2 \pi^{2}} \int_{-\infty}^{\infty} \frac{\mathrm{d} \tau}{\sqrt{1+\tau^{2}}} \int_{-\infty}^{\infty} \mathrm{d} \xi \frac{1}{\xi-x-y \tau} \frac{\partial}{\partial \xi} \mathcal{R}_{V} f(\xi, \tau)
$$

Now because of Equation (22), we get

$$
\begin{aligned}
f(x, y)= & -\frac{1}{2 \pi^{2}} \int_{-\infty}^{\infty} \frac{\mathrm{d} \tau}{\sqrt{1+\tau^{2}}} \int_{-\infty}^{\infty} \mathrm{d} \xi \frac{1}{\xi-x-y \tau} \frac{\partial}{\partial \xi} \mathcal{R}^{\natural} f(\xi, \tau) \\
& -\frac{1}{2 \pi^{2}} \int_{-\infty}^{\infty} \frac{\mathrm{d} \tau}{\sqrt{1+\tau^{2}}} \int_{-\infty}^{\infty} \mathrm{d} \xi \frac{1}{\xi-x-y \tau} \frac{\partial}{\partial \xi} \mathcal{R}^{\natural} f(\xi,-\tau) .
\end{aligned}
$$


The first line is just the reconstruction by $\mathcal{R}^{\natural}$ using the half-line Radon data $\mathcal{R}^{\natural} f(\xi, \tau)$, see Equation (19). So it is expected that the second integral on the data $\mathcal{R}^{\natural} f(\xi,-\tau)$ should vanish. This is the object of the next proposition

Proposition 4.4: $\mathcal{R}^{\natural} f(\xi,-\tau)$ belongs to the null space of the half-line reconstruction operator $\left(\mathcal{R}^{\natural}\right)^{-1}$

$$
\left(\mathcal{R}^{\natural}\right)^{-1} \mathcal{R}^{\natural} f(\xi,-\tau)=-\frac{1}{2 \pi^{2}} \int_{-\infty}^{\infty} \frac{\mathrm{d} \tau}{\sqrt{1+\tau^{2}}} \int_{-\infty}^{\infty} \mathrm{d} \xi \frac{1}{\xi-x-y \tau} \frac{\partial}{\partial \xi} \mathcal{R}^{\natural} f(\xi,-\tau)=0 .
$$

Proof: We use Equation (18) to replace $\mathcal{R}^{\natural} f(\xi,-\tau)$ by its defining integral. Then the left-hand-side of Equation (30) becomes

$$
-\frac{1}{2 \pi^{2}} \int_{-\infty}^{\infty} \frac{\mathrm{d} \tau}{\sqrt{1+\tau^{2}}} \int_{-\infty}^{\infty} \mathrm{d} \xi \frac{1}{\xi-x-y \tau} \frac{\partial}{\partial \xi} \sqrt{1+\tau^{2}} \int_{0}^{\infty} \mathrm{d} z f(\xi+z \tau, z) .
$$

Now insert the two-dimensional Fourier representation of $f(x, y)$

$$
f(x, y)=\int_{\mathbb{R}^{2}} \mathrm{~d} p \mathrm{~d} q \tilde{f}(p, q) e^{2 i \pi(p x+q y)},
$$

and use the well-known Fourier transform formula

$$
\int_{0}^{\infty} \mathrm{d} z e^{2 i \pi(p \tau+q) z}=\frac{1}{2}\left(\delta(p \tau+q)-\frac{1}{i \pi} \frac{1}{p \tau+q}\right),
$$

as well as

$$
\int_{-\infty}^{\infty} \frac{\mathrm{d} \xi}{\xi-(x+y \tau)}=e^{2 i \pi(x+y \tau)} i \pi \operatorname{sgn}(p)
$$

in Equation (31), to get a new form of $(31)^{2}$

$$
\begin{aligned}
& -\frac{1}{2 \pi^{2}} \int_{\mathbb{R}^{2}} \mathrm{~d} p \mathrm{~d} q \tilde{f}(p, q) e^{2 i \pi p x} i \pi \operatorname{sgn}(p) 2 i \pi p \\
& \times \int_{-\infty}^{\infty} \mathrm{d} \tau \frac{1}{2}\left(\delta(p \tau+q)-\frac{1}{i \pi} \frac{1}{p \tau+q}\right) e^{2 i \pi p y \tau} .
\end{aligned}
$$

The factor $2 i \pi p$ comes from the $\xi$ derivative of $\mathcal{R}^{\natural} f(\xi,-\tau)$. Note that $|p|=p \operatorname{sgn} p$, and $\delta(p \tau+q)=1 /|p| \delta(\tau+q / p)$. Performing the $\tau$ integral and using again Equation (32) give

$$
\frac{1}{2} f(x,-y)-\int_{\mathbb{R}^{2}} \mathrm{~d} p \mathrm{~d} q \tilde{f}(p, q) \frac{\operatorname{sgn}(p)}{2 i \pi} e^{2 i \pi x} \int_{-\infty}^{\infty} \mathrm{d} \tau \frac{e^{2 i \pi p y \tau}}{\tau+\frac{q}{p}},
$$

or using again

$$
\int_{-\infty}^{\infty} \mathrm{d} \tau \frac{e^{2 i \pi p y \tau}}{\tau+\frac{q}{p}}=e^{-2 i \pi q y} i \pi \operatorname{sgn}(p y),
$$

one ends up with the result

$$
\frac{1}{2}\{f(x,-y)-\operatorname{sgn}(y) f(x,-y)\}
$$


which is zero for $y>0$ as expected and equal to $f(x,-y)$ for $y<0$, also as expected.

Hence we have shown that

$$
\left(\mathcal{R}_{V}\right)^{-1}=\left(\mathcal{R}^{\natural}\right)^{-1}
$$

whenever these two inverse operators act on the data $\mathcal{R}_{V}$. Consequently, we deduce that symbolically

$$
\left(\mathcal{R}_{C C^{\prime}}\right)^{-1}=\left(\mathcal{R}_{C}\right)^{-1}
$$

with the corresponding provision on the scattering angle for each Radon transform

$$
\begin{aligned}
& \omega \in[\pi / 2, \pi] \text { for } \mathcal{R}_{C C^{\prime}} \\
& \omega \in[0, \pi] \text { for } \mathcal{R}^{\natural} .
\end{aligned}
$$

What can be concluded from this? If we count each $\mathcal{R}_{V} f(\xi, \tau)$ as one measurement in the $\mathcal{R}_{V}$-procedure (resp. each $\mathcal{R}^{\natural} f(\xi, \tau)$ as one measurement in the $\mathcal{R}^{\natural}$-procedure), then the number of measurements in the $\mathcal{R}_{V}$ procedure is just one half the number of measurements in the $\mathcal{R}^{\natural}$ procedure. This observation has an impact at the discretization level of the two inverse formulas as well as at the practical level of physical data collection. Thus discretized data shall appear as $\mathcal{R}^{\natural} f\left(\xi_{j}, \tau_{k}\right)$ and $\mathcal{R}_{V} f\left(\xi_{j^{\prime}}, \tau_{k^{\prime}}\right)$, where $(j, k)$ and $\left(j^{\prime}, k^{\prime}\right)$ are pairs of integers. The result of Theorem 4.3 means that the number of values taken by $k$ is twice the number of values taken by $k^{\prime}$. Consequently, this conclusion remains valid when transcribed to $\mathcal{R}_{C C^{\prime}}$ and to $\mathcal{R}_{C}$. This is the sense attributed to the 'improved' character given to a CST functioning with a pair of supplementary scanning circular arcs as compared to a CST functioning solely on a single scanning circular arc.

Remark 4.5: Equation (39) does not show that $\mathcal{R}_{V}$ is a pre-conditioned $\mathcal{R}^{\natural}$. If this were the case, then one should have

$$
\mathcal{R}_{V}=\mathcal{R}^{\natural} \mathcal{P}^{-1}
$$

where $\mathcal{P}$ is a non-singular pre-conditioner operator, lowering the condition number of $\mathcal{R}^{\natural}$. Of course the statement is to be understood at the discretized level, where each of the integral operator is replaced by its finite dimensional matrix representative. But this is not what Equation (40) shows.

\section{Numerical simulations}

30 As pointed out above, the inversion of the Radon transform on a pair of supplementary circular arcs $\mathcal{R}_{C} C^{\prime}$ is transferred to the inversion of the Radon transform on $V$-lines $\mathcal{R}_{V}$ by geometric inversion. So, in order to establish a numerical framework for practical reconstruction of functions, one must work first with the inverse formula of $\mathcal{R}_{V}$. The idea is to circumvent the divergent behaviour of the poles in Equation (17), by going to Fourier space to throw the divergence to infinity. But then one can 'apodize', as it is done currently with the Radon transform on full lines in the plane under the form of a FBP inversion method.

The reconstruction algorithm can be formulated as follows:

- From the two measurements at site $D$, work out the mathematical data $\mathcal{R}_{C C^{\prime}}(\xi, \omega)$ (given by Equations (14) and (3) as a function of $(\xi, \tau)$ ), for a real object defined by $f(x, y)$, 
- Use Equations (15) and (6) to generate mathematical data for $\mathcal{R}_{V}$ as a function of $\left(\xi^{\prime}, \tau^{\prime}\right)$, i.e. $\mathcal{R}_{C C^{\prime}} f(\xi, \omega)=\mathcal{R}_{V} g_{R}\left(\xi^{\prime}, \tau^{\prime}\right)$,

- Use formula (17) to perform the inversion of the $\mathcal{R}_{V}$ transform or to obtain the reconstruction of the 'apparent' object function

$$
\begin{aligned}
g_{R}\left(x^{\prime}, y^{\prime}\right)= & -\frac{1}{2 \pi^{2}} \int_{0}^{\infty} \frac{\mathrm{d} \tau^{\prime}}{\sqrt{1+\tau^{\prime 2}}} \\
& \times \int_{\mathbb{R}} \mathrm{d} \xi^{\prime}\left(\frac{1}{\xi^{\prime}-x^{\prime}-y^{\prime} \tau^{\prime}}+\frac{1}{\xi^{\prime}-x^{\prime}+y^{\prime} \tau^{\prime}}\right) \frac{\partial}{\partial \xi^{\prime}} \mathcal{R}_{V} g_{R}\left(\xi^{\prime}, \tau^{\prime}\right),
\end{aligned}
$$

- Rewrite this formula using the Fourier transforms

$$
\begin{aligned}
\mathcal{R}_{V} g_{R}\left(\xi^{\prime}, \tau^{\prime}\right) & =\int_{\mathbb{R}} \mathrm{d} q e^{2 i \pi q \xi^{\prime}} \widehat{\mathcal{R}_{V g_{R}}}\left(q, \tau^{\prime}\right), \\
\frac{1}{\xi^{\prime}-x^{\prime} \pm y^{\prime} \tau^{\prime}} & =i \pi \int_{\mathbb{R}} \mathrm{d} q^{\prime} e^{-2 i \pi q^{\prime}\left(\xi^{\prime}-x^{\prime} \pm y^{\prime} \tau^{\prime}\right)} \operatorname{sgn} q^{\prime} .
\end{aligned}
$$

We get, with $|q|=q \operatorname{sgn} q$,

$$
g_{R}\left(x^{\prime}, y^{\prime}\right)=\int_{\mathbb{R}} \frac{\mathrm{d} \tau^{\prime}}{\sqrt{1+\tau^{\prime 2}}} \int_{\mathbb{R}} \mathrm{d} q|q| \widehat{\mathcal{R}_{V} g_{R}}\left(q, \tau^{\prime}\right) e^{2 i \pi q x^{\prime}} 2 \cos \left(2 \pi q y^{\prime} \tau^{\prime}\right) .
$$

Recalling that $\tau^{\prime}=\cot \omega$, this reconstruction formula can be given the form of a FBP, as known in conventional Radon transform and also in [25],

- Recover the original object $f(x, y)$ using Equation (12).

- The term $|q|$ grows linearly at infinity. In order to approximate numerically this growth, one introduces a cut-off at the infinity bound (apodization).

\subsection{Projections}

The forward model given by (14) can be alternatively written in polar coordinates $(r, \theta)$ that 20 are more suitable for numerical integration on circular paths. Thus, keeping for readability the name of the function $f(r, \theta)$, we have

$$
\begin{aligned}
\mathcal{R}_{C C^{\prime}} f(\xi, \omega) & =I_{C C^{\prime}}(\rho, \omega) \\
& =\int_{0}^{\pi} \mathrm{d} \theta 2 \rho\left[f\left(2 \rho \cos \left(\theta-\omega+\frac{\pi}{2}\right), \theta\right)+f\left(2 \rho \cos \left(\theta+\omega-\frac{\pi}{2}\right), \theta\right)\right],
\end{aligned}
$$

25 that represents the integrations on two supplementary circular arcs of radius

$$
\rho=\frac{\xi}{2 \sin \omega} \text {. }
$$

Each scanning arc is linked to a scattering angle: either $\omega$ or $(\pi-\omega)$ (i.e. they are supplementary arcs of circle). For numerical simulations, we considered square $(N \times N$, $N=256)$ objects in the $(x, y)$ plane $(\Delta x=\Delta y=1)$ located at a distance $L=N$ of the source $S$ (i.e. the origin of coordinates, see Figure 6) and at a height $H_{0}=N / 4$ from 


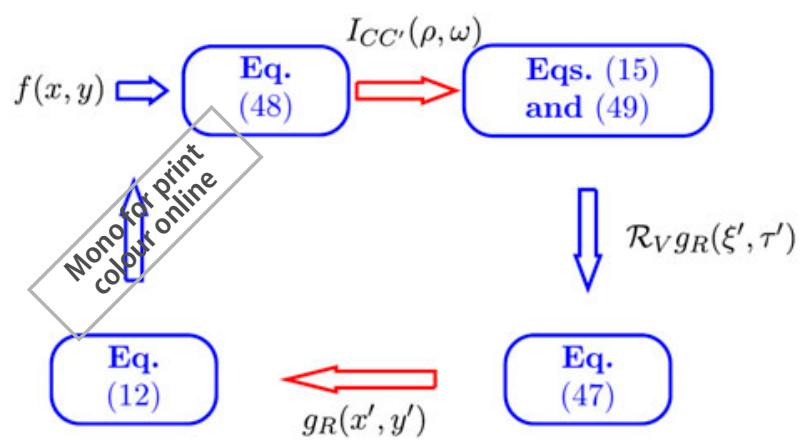

Figure 8. General reconstruction scheme for the modality CST on supplementary circle arcs.

the horizontal axis. The radius of the scanning arcs was discretized by $N \rho=2048$. The number of scattering angles considered was $N_{\omega}=2048$, with the parameter in $\omega \in\left(\frac{\pi}{2}, \pi\right)$ that corresponds to scattering angles in the full interval $(0, \pi)$. For the discretization of the polar angle we used $N_{\theta}=1024$ leading to an integration step $\Delta \theta=\frac{\theta_{\max }-\theta_{\min }}{N_{\theta}}$. The bounds of the interval are $0<\theta_{\max }, \theta_{\min }<\frac{\pi}{2}$ because the object considered was in the first quadrant. Simulated data is then used in reconstruction of functions.

\subsection{Reconstructions}

Reconstructions from data generated by (48) were performed according to the scheme outlined above and summarized in Figure 8. Equations (15) and (49) establish a link between $\mathcal{R}_{V} g_{R}\left(\xi^{\prime}, \tau^{\prime}\right)$ and $I_{C C^{\prime}}(\rho, \omega)$. Data obtained through (48) is rearranged in the form of $\mathcal{R}_{V} g_{R}\left(\xi^{\prime}, \tau^{\prime}\right)$ projections corresponding to the Radon transform on $V$-lines. For this purpose, geometric inversion was used with an inversion circle of radius $R=100$. The parameter $\xi^{\prime}$ (the distance to the origin) in the $\mathcal{R}_{V}$ projections was discretized as $\xi_{\min }^{\prime}=-90, \xi_{\max }^{\prime}=130$ and $N_{\xi^{\prime}}=2048$. The discretization of $\xi^{\prime}\left(N_{\xi^{\prime}}\right)$ and the scattering angle $\left(N_{\omega}=2048\right)$ provides enough data to reconstruct a $256 \times 256(N=256)$ object. After rearrangement, reconstruction is performed using filtered back-projection algorithm (47). In order to compensate the divergence of the ramp filter, we multiply by a cosine apodization window to obtain the final filter:

$$
W(q)=|q| \frac{1+\cos 2 \pi q}{2},
$$

where $q$ is the normalized frequency, dual to the variable $\xi^{\prime}$. The choice of this filter is motivated by the results obtained earlier in [26] where reconstruction from noisy and noiseless scatter radiation data was performed using a 3D filtered back-projection algorithm. In [26], several apodization windows (cosine, Shepp-Logan and Hamming) were tested and no significant differences between them were found. Finally, the desired reconstructions are obtained through (12), again with $R=100$.

\subsection{Results}

In order to illustrate the working of our new algorithm, we present numerical simulations applied on two objects: a cracked bar and a block of reinforced concrete. Each studied object 

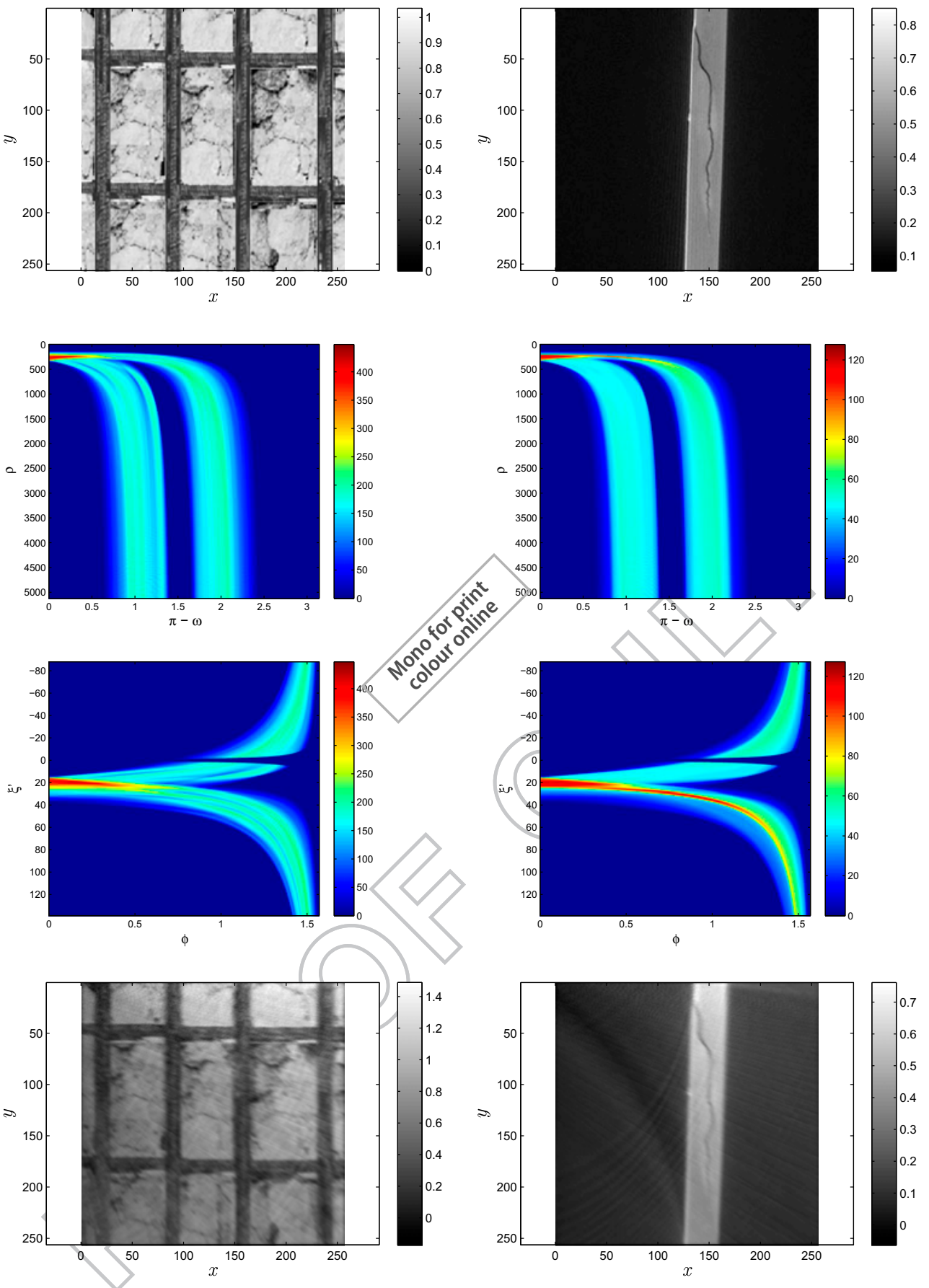

Figure 9. Radon Transform on supplementary circle arcs. Left column: reinforced concrete block. Right column: cracked bar. Row 1: original objects. Row 2: projection data: $I_{C C^{\prime}}(\rho, \omega)$. Row 3: Rearranged data as a $V$-line Radon Transform $\mathcal{R}_{V} g_{R}\left(\xi^{\prime}, \phi\right)\left(\tau^{\prime}=-\tan \phi\right)$. Row 4: reconstructions. See figures of merit in Table 1. 
Table 1. Normalized mean square error (\%).

\begin{tabular}{lcccr}
\hline & Noiseless & $20 \mathrm{~dB}$ & $15 \mathrm{~dB}$ & $10 \mathrm{~dB}$ \\
\hline Cracked bar & 0.41 & 0.47 & 0.59 & 0.97 \\
Reinforced concrete & 3.82 & 4.29 & 5.34 & 8.41 \\
\hline
\end{tabular}
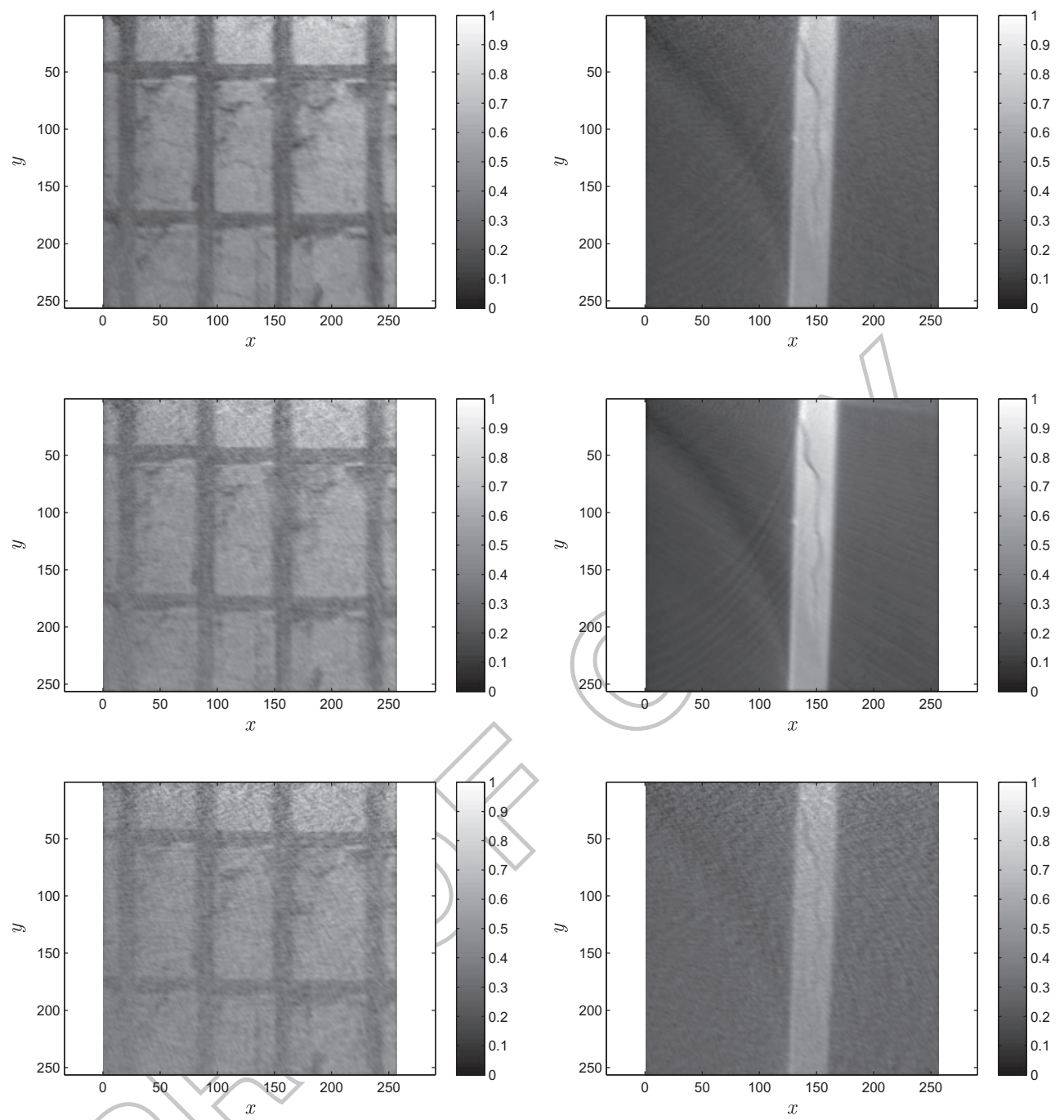

Figure 10. Reconstructions from noisy projections with the $\mathcal{R}_{C C^{\prime}}$. Row 1: $20 \mathrm{~dB}$. Row 2: $15 \mathrm{~dB}$. Row 3: 10 dB. See figures of merit in Table 1.

has different features that are useful to highlight the performance of the reconstruction technique applied. While the bar exhibits a thin structure, approximately $15 \%$ of the region of interest (ROI), with a homogeneous electronic density and a small detail in it (i.e. a crack), the block is a large massive-object made of different electronic densities (i.e. metal and concrete) and covering the whole ROI (100\%). Figure 9 shows the original objects 
together with projections $I_{C C^{\prime}}(\rho, \omega)$, rearranged data in the form of $V$-line projections (we show $\mathcal{R}_{V} g_{R}\left(\xi^{\prime}, \phi\right)=\mathcal{R}_{V} g_{R}\left(\xi^{\prime}, \tau^{\prime}\right)$ with $\tau^{\prime}=-\tan \phi$ and we kept the name $\mathcal{R}_{V} g_{R}$ for readability, and reconstructions. Notice the two branches in $\mathcal{R}_{C C^{\prime}} f(\xi, \omega)$, each one corresponding to a different circular path in (48). The missing data in $\mathcal{R}_{V} g_{R}\left(\xi^{\prime}, \phi\right)$ (in the neighbourhood of $\xi^{\prime}=0$ ) corresponds large scanning circumferences (i.e. $\rho$ large) and can be reduced by increasing $\rho$.

In addition, we study the robustness of the algorithm in front of noisy data. Projections calculated according previous section are now corrupted with Gaussian additive noise with three different signal to noise ratios (SNR): $20 \mathrm{~dB}, 15 \mathrm{~dB}$ and $10 \mathrm{~dB}$. Reconstructions were performed as in previous section and are shown in Figure 10. Finally, the quality of all reconstructions is assessed with the Normalized Mean Square Error [26,27] as a measurement of the accuracy of the method:

$$
\mathrm{NMSE}=100 \sum_{i=1}^{N^{2}}\left(\tilde{\mathbf{f}}_{\mathbf{i}}-\mathbf{f}_{\mathbf{i}}\right)^{2} /\left(N^{2} \max _{i}\left\{\mathbf{f}_{\mathbf{i}}\right\}\right),
$$

where vectors $\mathbf{f}$ and $\tilde{\mathbf{f}}$ are the reference and reconstructed functions, respectively. All results are summarized in Table 1.

\section{Discussion}

Results show a good performance of the inversion method proposed to reconstruct objects scanned with the CST modality on supplementary circle arcs. The crack in the bar is properly recovered, even in its thinnest part towards the bottom. The shape of the metal

20 bars inside the block of reinforced concrete is clearly seen. The main features of the non-metallic part are recovered although some blurring is present in the smallest details. Regarding figures of merit, the obtained NMSEs are reasonable. In the case of the bar (NMSE $=0.41 \%$ ), the most contributing factor is the non-zero background (see Figure 9) present in the original image that is slightly amplified in the reconstruction. Nevertheless, this does not alter significantly the appearance of the reconstructed image. On the other hand, inside the reinforced concrete block, the error (NMSE $=3.82 \%$ ) is completely due to the object itself since there is not background. Missing data in $\mathcal{R}_{V}$ does not seem to be critical for reconstructions. In what concerns noisy data $(\mathrm{SNR}=20 \mathrm{~dB}$ and $15 \mathrm{~dB})$, although there is some degradation in the quality of the recovered images, the small structures or the important details of the objects (i.e. the crack in the bar and the state of the metal skeleton in the reinforced concrete) are well observed even without using any de-noising technique. With $\mathrm{SNR}=10 \mathrm{~dB}$ or lower some previous treatment may be necessary.

\section{Conclusion and perspectives}

In this paper, we have introduced a new modality of CST which is suitable for one-sided large objects located in a half space in $\mathbb{R}^{3}$. This corresponds to the growing need of imaging objects of interest in those fields cited in Section 1. It consists in 'doubling' the Compton effect scanning of the sample as compared to the 'single' of the conventional modality of CST. Theoretically, image reconstruction should be of enhanced quality as 
discussed in Section 4. Numerical simulations results support this aspect of the new CST and open the way to further research development directions taking into account attenuation corrections, photometric propagation effects as well as non-isotropic, nonmonochromatic source before it can become as a well-established imaging procedure for wide use in the fields mentioned.

\section{Notes}

1. S. J. Norton has also worked out a related inversion formula based on the result of A. M. Cormack.

2. Here $\operatorname{sgn}(p)$ is the sign function of $p$.

\section{Acknowledgements}

The authors would like to thank all the referees for their critical remarks and suggestions which have contributed to the improvement of this paper.

\section{Disclosure statement}

15 No potential conflict of interest was reported by the authors.

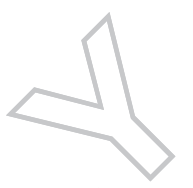

\section{Funding}

J. Cebeiro research work is supported by a CONICET [grant number Nro. Legajo 146618]. The work of J. Cebeiro and M. A. Morvidone is partially supported by SOARD-AFOSR [grant number FA955014-1-0276]. J. Cebeiro acknowledges support from Programa BEC.AR for financial funding of a visit to ETIS (ENSEA/ Université de Cergy-Pontoise/UMR CNRS 8051) in 2015. M. A. Morvidone was partially supported by MINCyT-CONICET-CNRS (International Cooperation Programme, CONICET Resol. 147/15).

\section{References}

[1] Shengli N, Jun Z, Liuxing H. EGS4 simulation of Compton scattering for nondestructive testing. In: Proceedings of the 2nd International Workshop on EGS, Tsukuba Japan 2000. KEK Proceedings. 2000;200(20):216-223.

[2] Gerl J. Gamma-ray imaging exploiting the Compton effect. Nucl Phys A. 2005;752:688c-695c.

[3] Abdul-Majid S, Balamesh A. Imaging corrosion under insulation by gamma ray backscattering method. In: 18th World Conference on Nondestructive Testing; 2012 Apr 16-20; Durban, South Africa.

[4] Truong TT, Nguyen MK. Recent developments in Compton scatter tomography: theory and numerical simulations. In: Andriychuk M, editor. Numerical simulation: from theory to industry. Croatia: INTECH Rijeka; 2012. p. 101-128.

[5] Harding G, Harding E. Compton scatter imaging: a tool for historical exploration. Appl Radiat Isot. 2010;68:993-1005.

[6] Hussein EMA, Desrosiers M, Waller EJ. On the use of radiation scattering for the detection of landmines. Radiat Phys Chem. 2005;73:7-19.

[7] Cruvinel PE, Balogun FA. Compton scattering tomography for agricultural measurements. Eng Agr Jaboticabal. 2006;26(1):151-160.

40 [8] Itou M, Orikasa Y, Gogyo Y, et al. Compton scattering imaging of a working battery using synchrotron high-energy X-rays. J Synchrotron Radiat. 2015;22:161-164. 
[9] Lange A, Hentschel MP, Kupsch A, et al. X-ray Compton tomography. In: 11th European Conference on Non-Destructive Testing (ECNDT 2014); 2014 Oct 6-10; Prague, Czech Republic.

[10] Adejumo OO, Balogun FA, Egbedokun GGO. Developing a Compton scattering tomography system for soil studies: theory. J Sustain Dev Environ Prot. 2011;1(3):73-81.

[11] Thoe RS. Single-sided tomography of extremely large dense objects. In: Proceedings of SPIE 1942, Underground and Obscured Object Imaging and Detection, Vol. 289; 1993 Nov 15.

[12] Norton SJ. Compton scattering tomography. J Appl Phys. 1994;76(4):2007-2015.

[13] Truong TT, Nguyen MK. New properties of the V-line Radon transforms and their imaging applications. J Phys A Math Theor. 2015;48:405204.

[14] Cormack AM. Representation of a function by its line integrals, with some radiological applications. J Appl Phys. 1964;34(9):2722-2727.

[15] Florescu L, Markel V, Schotland JC. Inversion formulas for the broken-ray Radon transform. Inverse Prob. 2011;27:025002.

[16] Gautam SR, Hopkins FF, Klinksiek R, et al. Compton interaction tomography. IEEE Trans Nucl Sci. 1983;NS-30:1680-1684.

[17] Novikov RG. An inversion formula for the attenuated X-ray transform. Ark Math. 2002;40:145167.

[18] Rigaud G, Nguyen MK, Louis AK. Novel numerical inversions of two circular-arc radon transforms in Compton scattering tomography. Inverse Prob Sci Eng. 2012;20:809-839.

[19] Barrett HH. The Radon transform and its applications. In: Wolf E, editor. Progress in optics. Vol. 21. North Holland; 1984. p. 219-286.

[20] Truong TT, Nguyen MK. On new V-line Radon transforms in R2 and their inversion. J Phys A Math Theor. 2011;44:075206.

[21] Amabartsoumain G, Moon S. A series formula for inversion of the V-line Radon transform in a disc. Comput Math Appl. 2013;66:1567-1572.

[22] Katsevich A, Krylov R. Broken ray transform: inversion and a range condition. Inverse Prob. 2013;29:075008.

[23] Ilmairta J. Broken ray tomography in the disc. Inverse Prob. 2013;29:035008.

30 [24] Zhao F, Schotland JC, Markel VA. Inversion of the star transform. Inverse Prob. 2014;30:105001.

[25] Morvidone M, Nguyen MK, Truong TT, et al. On the V-line Radon transform an its imaging applications. Int J Biomed Imaging. 2010;2010:208179.

[26] Cebeiro J, Morvidone MA, Nguyen MK. Back-projection inversion of a Conical Radon transform. Inverse Prob Sci/Eng. 2015;24(2):328-352.

[27] Cebeiro J, Lebailly Q, Morvidone MA, et al. A new modality of bidimensional Compton camera. In: Proceedings of IEEE 37th Annual International Conference of the Engineering in Medicine and Biology Society (EMBC'2015). Milano, Italy; 2015. p. 6301-6304. 